\title{
Adaptabilidade e estabilidade de populações de cenoura pelo método REML/BLUP
}

\author{
Agnaldo DF Carvalho; Giovani O Silva; Francisco V Resende
}

Embrapa Hortaliças, Brasilia-DF, Brasil; agnaldo.carvalho@embrapa.br; giovani.olegario@embrapa.br; francisco.resende@embrapa.br

\begin{abstract}
RESUMO
O objetivo foi avaliar a adaptabilidade e estabilidade de genótipos de cenoura pelo método REML/BLUP. Os experimentos foram conduzidos em 20 ambientes nos anos de 2010, 2011, 2012 e 2014 em diferentes regiões produtoras de cenoura do Brasil. O delineamento experimental utilizado foi de blocos ao acaso com três repetições e parcelas de $1 \mathrm{~m}^{2}$. Foram avaliadas quatro populações de cenoura em fase de melhoramento: CNPH-14, CNPH-15, CNPH-16 e CNPH17, as cultivares de polinização aberta BRS Planalto e Brasília e o hibrido Juliana. Aos 100 dias após a semeadura foi determinado em cada parcela o rendimento de raízes com padrão comercial. Foi utilizada a metodologia REML/BLUP para estimar a adaptabilidade e estabilidade dos genótipos. Pôde-se verificar que as populações com melhor desempenho e adaptabilidade e estabilidade nos ambientes estudados foram BRS Planalto, CNPH-14 e CNPH-17. A cultivar Juliana e a população CNPH-15 apresentaram os piores resultados frente à média dos ambientes, e Juliana apresentou adaptação específica a São Gotardo-MG e Canoinhas-SC. As populações CNPH-14 e CNPH-17 possuem adaptação ampla o que facilita a recomendação como futuras cultivares.
\end{abstract}

Palavras-chave: Daucus carota, interação genótipos x ambientes, produção comercial.

\section{ABSTRACT \\ Adaptability and stability of carrot populations by the REML/ BLUP method}

The aim was to evaluate the adaptability and stability of the genotypes by REML/BLUP method. The experiments were carried out in 20 environments in the years 2010, 2011, 2012 and 2014 in different carrot producing regions in Brazil. The experimental design was a randomized block design with three replications and plots of 1 $\mathrm{m}^{2}$. Four carrot populations in breeding phase were evaluated: CNPH14, CNPH-15, CNPH-16 and CNPH-17, the open-pollinated cvs. BRS Planalto and Brasília and the hybrid Juliana. At 100 days after sowing we determined in each plot the root yield with commercial standard. We used the REML/BLUP methodology to estimate the adaptability and stability of genotypes. We observed that populations with better performance and adaptability and stability in the study sites were the populations CNPH-14 and CNPH-17. The cv. Juliana and the population CNPH-15 showed the worst results compared to the average of the environment, and Juliana had specific adaptation to São Gotardo, Minas Gerais State and Canoinhas, Santa Catarina State, Brazil. The populations CNPH-14 and CNPH-17 have broad adaptation which facilitates the recommendation as future cultivars.

Keywords: Daucus carota, genotype x environment interaction, commercial production.

(Recebido para publicação em 26 de março de 2015; aceito em 7 de junho de 2016) (Received on March 26, 2015; accepted on June 7, 2016)

\begin{abstract}
A cenoura é uma hortaliça da família Apiaceae, do grupo das raízes tuberosas, cultivada em larga escala nas regiões Centro-Oeste, Sudeste, Nordeste e Sul do Brasil. A área plantada no Brasil é cerca de 24,5 mil hectares com produção de 760 mil toneladas de raízes (Anuário Brasileiro de Hortaliças, 2015). Embora produza melhor em áreas de clima ameno, nos últimos anos, em virtude do desenvolvimento de cultivares tolerantes ao calor, o plantio de cenoura vem-se expandindo para regiões mais quentes do país.

Para o lançamento de novas culti-
\end{abstract}

vares de cenoura, conhecer o comportamento desses genótipos em relação aos ambientes onde os mesmos possam ser indicados para plantio, é de fundamental importância para o melhorista (Silva et al., 2012). O comportamento de um genótipo em relação às variações ambientais pode ser estudado através das análises de estabilidade e adaptabilidade.

A expressão fenotípica de um caráter é o resultado do efeito do genótipo (G), do ambiente (A) e da interação entre esses efeitos o que é denominado interação GxA. O fator ambiental é o responsável pela não coincidência de um mesmo genótipo cultivado em diferentes ambientes (Cruz et al., 2012). Assim, é comum a existência de cultivares que se adaptam a uma maior amplitude de ambientes enquanto outras possuem adaptação restrita aos locais onde são indicadas para cultivo. A presença da interação GxA é parte fundamental em programas de melhoramento, uma vez que interfere na recomendação das cultivares. Uma cultivar ideal seria aquela com adaptação em todos os ambientes de cultivo. Contudo, a situação mais comum são cultivares sendo recomen- 
dadas para locais ou regiões onde as mesmas possuem maior estabilidade de produção e maior adaptabilidade àquele ambiente (Campbell \& Jones, 2005).

Conforme descrito por Cruz et al. (2012), a adaptabilidade e estabilidade são muito importantes em programas de melhoramento genético. O primeiro refere-se à capacidade dos genótipos se beneficiarem ao estímulo do ambiente, enquanto a estabilidade denota o comportamento previsível do genótipo frente a mudanças ambientais. Muitas metodologias têm sido propostas para estudar a adaptabilidade e estabilidade, sendo exemplos às metodologias que utilizam análise de variância (Wricke, 1965), análise de regressão (Eberhart \& Russel, 1966) e análise não paramétrica (Lin \& Binns, 1988). No entanto, observa-se que, apesar dos algoritmos tradicionais terem ajudado muito na seleção dos genótipos mais adaptados e estáveis, muitas vezes, os mesmos, não têm explicado de forma satisfatória o comportamento de genótipos de várias espécies (Duarte \& Zimmerman, 1994; Osmanzai \& Sharma, 2008; Namorato et al., 2009; Silva et al. 2011, 2012). Uma alternativa mais recente que procura deixar os resultados mais fáceis de serem interpretados e com informações mais práticas tem sido a utilização das equações de modelos mistos REML/ BLUP (Máxima Verossimilhança Residual ou Restrita/Melhor Predição Linear Não Viesada).

A análise REML/BLUP baseia-se nos seguintes estimadores: quanto menor o desvio-padrão do valor genotípico nos locais de avaliação, maior será a média harmônica dos valores genotípicos nos mesmos ambientes avaliados. Desse modo, a seleção dos maiores valores genotípicos das médias harmônicas (MHVG) consegue simultaneamente selecionar os indivíduos com maior produtividade e estabilidade. A adaptabilidade refere-se ao desempenho relativo dos valores genotípicos (PRVG) nos ambientes. Dessa forma, os valores genotípicos preditos (ou os dados originais) são representados como proporção da média geral de cada ambiente e, em seguida, obtém-se o valor médio dessa proporção através dos locais. A seleção conjunta para produção, estabilidade e adaptabilidade, utilizando modelos mistos, é feita pelo método da média harmônica do desempenho relativo dos valores genéticos (MHPRVG) preditos. Esse método, além de selecionar ao mesmo tempo pelas três propriedades mencionadas, apresenta as seguintes vantagens: (a) considera os efeitos aleatórios e, portanto estima a estabilidade e adaptabilidade genotípica e não fenotípica; (b) elimina a pressuposição de balanceamento; (c) os delineamentos não precisam necessariamente ser ortogonais; (d) admite desigualdades de variâncias; (e) considera erros correlacionados dentro de locais; (f) elimina os efeitos de instabilidade dos valores genéticos; (g) não existe limitação quanto ao número máximo e mínimo de ambientes; (h) considera a estabilidade e adaptabilidade na seleção de indivíduos dentro da população; (i) independe da estimava de outros parâmetros como os coeficientes de regressão; (j) resultados são padronizados com as do caráter avaliado; (k) possibilita fazer a seleção levando em conta três atributos simultaneamente (Resende, 2002a).

Silva et al. (2011) compararam os métodos GGE Biplot, AMMI e REML/ BLUP para verificação da adaptabilidade e estabilidade de populações de cenoura e verificaram vantagens desta última metodologia, por apresentar os resultados em função de valores genotípicos levando em consideração os parâmetros de adaptabilidade e estabilidade.

Desta forma, em função das razões e da flexibilidade dos métodos descritos acima, o objetivo desse estudo foi avaliar a adaptabilidade e estabilidade de genótipos de cenoura pelo método REML/BLUP.

\section{MATERIAL E MÉTODOS}

O presente estudo foi conduzido nos anos de 2010, 2011, 2012 e 2014. Em 2010 e 2011 os experimentos foram realizados em São Gotardo-MG, Gama-DF e Lapão-BA em sistema de cultivo convencional e em Gama, Brazlândia-DF e Comunidade Agrícola Lamarão-DF, em cultivo orgânico. Em 2012 os cultivos foram realizados no Gama, São Gotardo e Canoinhas-SC em sistema convencio- nal e no Gama e Brazlândia em cultivo orgânico. Em 2014 os cultivos foram realizados no Gama em sistema orgânico e convencional e em Taguatinga-DF em sistema orgânico.

O delineamento experimental utilizado em todos os 20 ambientes foi de blocos ao acaso com três repetições. Foram avaliadas quatro populações de cenoura do grupo Brasília: CNPH-14, CNPH-15, CNPH-16 e CNPH-17 e duas cultivares de polinização aberta: BRS Planalto, Brasília e o hibrido Juliana. As parcelas constituíram-se de $1 \mathrm{~m}^{2}$, com 6 linhas em espaçamento duplo, sendo a distância entre linhas duplas de $20 \mathrm{~cm}$, entre linhas simples de $10 \mathrm{~cm}$ e entre plantas de $5 \mathrm{~cm}$. O manejo de campo foi realizado conforme as práticas utilizadas em cada propriedade.

As colheitas foram realizadas aos 100 dias após a semeadura e foi determinada em cada parcela, em gramas, a massa fresca das raízes comercializáveis de acordo com o padrão proposto pela Ceagesp (1999), não sendo considerado como raízes comerciais as que apresentaram diâmetro do terço médio superior inferior a $2 \mathrm{~cm}$.

A avaliação da adaptabilidade e estabilidade foi realizada por meio da metodologia REML/BLUP(Henderson, 1975), considerando o seguinte modelo estatístico:

$$
Y=X_{r}+Z_{g}+W_{i}+e
$$

Em que: $Y$ é o vetor de dados, $r$ é o vetor dos efeitos de repetição (assumidos como fixos) somados à média geral, e contemplam todas as repetições de todos os locais, g é o vetor dos efeitos genotípicos (assumidos como aleatórios), i é o vetor dos efeitos da interação genótipos $\mathrm{x}$ locais (aleatórios), sendo e o vetor de erros (aleatórios). As letras maiúsculas representam as matrizes de incidência para os referidos efeitos.

As equações do modelo misto para se equivalerem a predição de r, g e i são:

$$
\left[\begin{array}{ccc}
X^{\prime} X & X^{\prime} Z & X^{\prime} W \\
Z^{\prime} X & Z^{\prime} Z+\lambda_{1} & Z^{\prime} W \\
W^{\prime} X & W^{\prime} Z & W^{\prime} W+\lambda_{2}
\end{array}\right]\left[\begin{array}{c}
\hat{r} \\
\hat{g} \\
\hat{\imath}
\end{array}\right]=\left[\begin{array}{c}
X^{\prime} y \\
Z^{\prime} y \\
W^{\prime} y
\end{array}\right],
$$

Em que:

$$
\lambda_{1}=\frac{1-h_{g}^{2}-h_{i}^{2}}{h_{g}^{2}} ; \lambda_{2}=\frac{1-h_{g}^{2}-h_{i}^{2}}{h_{i}^{2}} ;
$$


Em que: $\mathrm{h}_{\mathrm{g}}^{2}=$ herdabilidade individual, no sentido amplo em nível de bloco; $h_{i}^{2}=$ coeficientes de determinação dos efeitos da interação genótipos $\mathrm{x}$ ambientes.

Para avaliação genética pelos maiores valores da média harmônica dos valores genotípicos foi utilizado o método (MHPRVG); conforme descrito por Resende (2002a):

$$
\text { MHPRVG }=\frac{n}{\sum_{j=1}^{n} \frac{1}{V g_{i j}}}
$$

Em que: n é o número de locais onde se avaliou o genótipo i; e Vgij é o valor genotípico do genótipo i no local j, expresso como proporção da média desse local.

Para a realização das análises foi utilizado o aplicativo computacional Selegen (Resende, 2002b).

\section{RESULTADOS E DISCUSSÃO}

A análise de deviance conjunta para a massa fresca de raízes comercializáveis revelou os efeitos de populações como não significativos (Tabela 1). Já a interação entre populações e ambientes foi significativa, indicando que as populações apresentaram respostas diferenciadas quando submetidas aos diferentes ambientes (locais e anos) de cultivo. Isto revela que ocorreu alteração no posicionamento das populações ou mudanças na magnitude das diferenças entre as populações e os ambientes estudados, o que pode ser confirmado pelo reduzido valor da correlação genética entre ambientes (rgloc), que foi de $0,06 \%$.

O coeficiente de variação foi de $17,41 \%$ conferindo boa precisão ao experimento, e alta confiabilidade das estimativas obtidas, pois massa de raiz é um caráter altamente influenciado pelo ambiente (Vieira \& Silva, 2008; Vieira et al., 2009, Silva et al., 2012). Este valor se aproximou aos observados por Oliveira et al. (2005) e Silva et al. (2012) e abaixo daqueles verificados por Oliveira et al. (2008).

A média de rendimento de raízes comercializáveis no sistema convencional de cultivo foi cerca de $30 \%$ superior

Tabela 1. Valores da estatística do teste razão de verossimilhança (LRT) da análise de deviance conjunta, média geral e coeficiente de variação experimental (CV\%) para massa fresca de raiz comercial de populações de cenoura avaliadas em cinco locais, nas safras agrícolas 2007/2008, 2008/2009 e 2009/2010 \{values of the statistical likelihood ratio test (LRT) of the joint deviance analysis, general average and coefficient of experimental variation (CV\%) for fresh mass of marketable roots of carrot populations tested at five locations, in the 2007/2008, 2008/2009 and 2009/2010 crops\}. Brasília, Embrapa Hortaliças, 2014.

\begin{tabular}{lc}
\hline Efeitos $^{1}$ & Massa fresca de raiz comercial \\
\hline Populações & $1,16^{\text {ns }}$ \\
Populações x Ambientes & $38,33^{*}$ \\
\hline CV $(\%)$ & 17,41 \\
Média geral & 34,76 \\
Média do sistema convencional & 40,03 \\
Média do sistema orgânico & 29,50 \\
\hline
\end{tabular}

${ }^{1}$ Valores de LRT; Significativo a $* \mathrm{P}=0,05$ e ${ }^{\text {ns }}$ não significativo pelo teste $\chi 2$ com 1 grau de liberdade. ( ${ }^{1}$ LRT values, *Significant $\mathrm{p}=0.05$ and not significant by $\chi 2$ test with one degree of freedom).

ao sistema orgânico. Estes resultados são concordantes com os obtidos no estudo de Carvalho et al. (2005) que observaram valor $22 \%$ maior no sistema convencional para este mesmo caráter. Estes autores justificaram o menor rendimento em sistema orgânico devido ao fato daquele campo, em particular, ainda estar em processo de construção da fertilidade pela adição dos compostos orgânicos, adubos verdes e fosfatos naturais. No presente estudo, alguns sistemas orgânicos se destacaram, por exemplo, GAO14 que apresentou médias elevadas para todos os genótipos avaliados, pois trata-se de um sistema orgânico de produção já construído, corroborando com os resultados de Souza (1996) que observou produtividade de cenoura 7\% superior no sistema de cultivo orgânico em relação ao convencional, em estudos realizados durante dez anos. O que contribuiu para a redução da média do sistema orgânico, no presente trabalho, foram ambientes como GAO10 e BRO10 que, por estarem em processo de construção de fertilidade, acabaram refletindo negativamente nas médias dos genótipos.

$\mathrm{Na}$ Tabela 2 é possível visualizar as populações com os respectivos valores genotípicos somados às médias para cada ambiente e em conjunto para todos os ambientes de cultivo. Pode-se verificar que a população 15 e a cultivar Juliana tiveram os piores desempenhos genotípicos médios no conjunto de am- bientes. Os melhores genótipos foram as populações CNPH-14, CNPH-17, e a cv. BRS Planalto, com médias superiores à média geral do experimento 34,76 t/ha.

Os maiores rendimentos foram verificados em São Gotardo nos anos 2010 e 2011, em Gama em 2014 e em Canoinhas em 2012, com rendimentos chegando a 82,56 t/ha para a cv Juliana em Canoinhas. A região de São Gotardo se caracteriza por elevados rendimentos devido ao alto investimento em insumos como irrigação, adubação, agroquímicos e cultivares híbridas. Nesta mesma região, nos três anos de cultivo, a cv. Brasília esteve entre as populações com maiores rendimentos, variando de 43,97 a 50,77 t/ha. Brasília foi a cultivar responsável pelo desenvolvimento da cultura da cenoura naquela região, ocupando quase a totalidade da área plantada até 2004. Após este período, com o lançamento de cultivares híbridas, com melhor qualidade de raiz, coloração mais alaranjada e melhor aparência, naturalmente, Brasília deixou de ser cultivada.

Apesar da média nacional de produção ser de 31,02 t/ha (Anuário Brasileiro de Hortaliças, 2015), alguns estudos indicam a possibilidade de, em condições ideais de manejo, ser possível alcançar produtividades semelhantes às máximas obtidas neste trabalho. Resende \& Braga (2014) verificaram produtividade de raízes comercializáveis máximas de 81,70 t/ha para a cv. Brasília, e de 
Tabela 2. Valores genotípicos pela metodologia REML/BLUP somados à media, para massa fresca de raízes comercializáveis de populações de cenoura avaliadas em 20 ambientes, nos anos de 2010, 2011, 2012 e 2014 (genotypic values generated by the methodology REML/BLUP plus the mean for fresh mass of marketable roots of carrot populations tested in 20 environments, in 2010, 2011, 2012 and 2014 years). Brasília, Embrapa Hortaliças, 2014.

\begin{tabular}{lccccccc}
\hline Local & \multicolumn{7}{c}{ Populações de cenoura } \\
\cline { 2 - 7 } & CNPH-14 & CNPH-15 & CNPH-16 & CNPH-17 & Brasília & BRS Planalto & Juliana (F $)$ \\
\hline SGC101 & 49,72 & 43,25 & 48,19 & 51,87 & 50,77 & 48,81 & 51,85 \\
GAC10 & 25,42 & 17,83 & 24,51 & 26,68 & 21,39 & 29,03 & 17,90 \\
GAO10 & 14,36 & 10,10 & 11,92 & 15,36 & 14,73 & 13,23 & 11,74 \\
BRO10 & 16,90 & 14,61 & 17,24 & 18,48 & 16,23 & 17,11 & 14,89 \\
LMO10 & 23,34 & 24,85 & 25,49 & 27,78 & 24,16 & 29,10 & 23,22 \\
LAC10 & 27,89 & 29,24 & 30,22 & 32,65 & 33,17 & 32,06 & 31,87 \\
GAC11 & 24,07 & 24,56 & 20,74 & 23,68 & 20,74 & 22,33 & 22,14 \\
GAO11 & 38,57 & 34,11 & 37,62 & 38,83 & 38,30 & 37,24 & 32,38 \\
LAC11 & 31,63 & 28,75 & 27,83 & 30,77 & 34,70 & 32,31 & 30,40 \\
LMO11 & 20,86 & 20,60 & 23,92 & 20,92 & 16,79 & 18,57 & 19,52 \\
BRO11 & 18,97 & 20,36 & 21,29 & 21,39 & 16,71 & 22,52 & 17,90 \\
SGC11 & 43,98 & 44,86 & 45,21 & 42,61 & 43,97 & 41,91 & 40,68 \\
BRO12 & 25,20 & 21,82 & 25,18 & 28,40 & 27,26 & 22,40 & 22,00 \\
CAC12 & 80,31 & 72,07 & 56,94 & 74,88 & 61,22 & 80,52 & 82,56 \\
GAC12 & 31,63 & 30,10 & 29,97 & 33,20 & 34,89 & 26,48 & 22,72 \\
GAO12 & 35,94 & 36,63 & 36,31 & 41,29 & 41,07 & 40,90 & 42,22 \\
SGC12 & 41,45 & 43,10 & 45,33 & 45,61 & 47,95 & 43,95 & 50,97 \\
GAC14 & 52,99 & 48,10 & 53,02 & 55,14 & 47,30 & 55,79 & 51,84 \\
GAO14 & 71,66 & 67,90 & 72,81 & 75,52 & 70,46 & 74,40 & 65,65 \\
TAO14 & 29,57 & 26,49 & 29,46 & 28,42 & 29,00 & 30,42 & 24,74 \\
\hline MCon & 40,91 & 38,19 & 38,20 & 41,71 & 39,61 & 41,32 & 40,29 \\
MOrg & 29,54 & 27,75 & 30,12 & 31,64 & 29,47 & 30,59 & 27,43 \\
Média & 35,22 & 32,97 & 34,16 & 36,67 & 34,54 & 35,95 & 33,86 \\
\hline Asd & 29 &
\end{tabular}

${ }^{1}$ As duas primeiras letras representam o local de cultivo, a terceira o sistema de cultivo e os dois dígitos os anos de colheitas dos experimentos (the first two letters represent the place of cultivation, the third the cultivation system and the two digits the harvest year of the experiments); $\mathrm{SG}=$ São Gotardo-MG, GA=Gama-DF, BR=Brazlândia-DF, LM=Lamarão-DF, LA=Lapão-BA; CA=Canoinhas-SC; TA= Taguatinga-DF; O= Sistema orgânico (organic system); C= Sistema convencional (conventional system), 10= Safra (crop season) 2009/10; 11= Safra 2010/11; 12= Safra=2011/12 e 14=Safra 2013/14; MCon= média dos ambientes de cultivo convencional (average in the conventional system), MOrg= média dos ambientes de cultivo orgânico (average in the organic system).

84,50 t/ha para uma população derivada de Brasília. Corroborando com esse potencial, Resende \& Cordeiro (2007) e Soares et al. (2010) obtiveram rendimentos próximos a $80 \mathrm{t} / \mathrm{ha}$ com essa mesma cultivar.

A população CNPH-17 destacou-se positivamente, estando entre aquelas com maiores médias corrigidas pelos valores genotípicos para praticamente todos os ambientes. Em seguida, a população CNPH-14 também apresentou valores altos para a maioria dos ambientes. As cultivares Brasília e BRS Planalto e a população CNPH-16 tiveram valores superiores em cerca de metade dos ambientes e a população CNPH-15 e a cultivar Juliana foram aquelas que se destacaram no menor número de ambientes.

O fato da cultivar Juliana ter se destacado em São Gotardo e Canoinhas mas não na maioria dos outros ambientes, indica que a mesma é adaptada para ambientes sem restrição na utilização de insumos, ou seja, é responsiva em ambientes férteis, bem irrigados e sem a interferência de doenças foliares, que são controladas com agroquímicos.

Chama a atenção a população
CNPH-17 pelo bom desempenho médio, tanto nos sistemas orgânico (31,64 t/ha), como convencional (41,71 t/ha), o que possivelmente justifique o lançamento dessa população como cultivar adaptada a esses dois sistemas de cultivo. Essa capacidade já foi relatada por Silva et al. (2012) ao avaliar duas populações junto com a cultivar Brasília em sistema orgânico e convencional e verificar desempenho semelhante das mesmas nos dois sistemas, ou seja, existindo genótipos com adaptação a diferentes sistemas de cultivo.

As cultivares Brasília e BRS Planal- 
Tabela 3. Estabilidade de valores genotípicos (MHVG), adaptabilidade de valores genotípicos (PRVG), estabilidade e adaptabilidade de valores genotípicos (MHPRVG) para o caráter massa de raízes comercializáveis de populações de cenoura avaliadas em 20 ambientes, nos anos de 2010, 2011, 2012 e 2014 \{stability of genotypic values (MHVG), adaptability of genotypic values (PRVG), stability and adaptability of genotypic values (MHPRVG) for the character mass of marketable roots of carrot genotypes tested in 20 environments, in 2010, 2011, 2012 and 2014\}. Brasília, Embrapa Hortaliças, 2014.

\begin{tabular}{lccc}
\hline \multirow{2}{*}{ População de cenoura } & \multicolumn{3}{c}{ Valores genotípicos } \\
\cline { 2 - 4 } & MHVG & PRVG & MHPRVG \\
\hline CNPH-14 & 28,91 & 35,25 & 35,11 \\
CNPH-15 & 26,22 & 32,74 & 32,50 \\
CNPH-16 & 28,40 & 34,64 & 34,41 \\
CNPH-17 & 30,65 & 37,06 & 36,97 \\
Brasília & 28,19 & 34,77 & 34,43 \\
BRS Planalto & 29,18 & 35,89 & 35,66 \\
Juliana ( $\left.\mathrm{F}_{1}\right)$ & 26,23 & 33,03 & 32,67 \\
\hline
\end{tabular}

to apresentaram o melhor desempenho em Lapão. Resultados semelhantes foram reportados por Silva et al. (2012) na avaliação dessas duas cultivares na mesma região ao verificar que as mesmas foram superiores a duas outras populações avaliadas. $\mathrm{O}$ bom desempenho de Brasília em Lapão responde o sucesso dessa cultivar e suas derivações, nessa região, pois além de adaptada e estável, possui boa produtividade, além de resistência à queima das folhas.

Na Tabela 3 estão apresentados os resultados esperados de raízes comercializáveis em t/ha, penalizando ou capitalizando as populações de acordo com seu desempenho em relação à estabilidade (MHVG), adaptabilidade (PRVG), e estabilidade e adaptabilidade conjuntamente (MHPRVG) para o conjunto de ambientes. Tanto em relação à estabilidade quanto para adaptabilidade, verifica-se que as populações CNPH-14, CNPH-17 e a cultivar BRS Planalto foram superiores aos demais genótipos, concordando com as estimativas de médias corrigidas pelos valores genotípicos apresentados. Os genótipos com menor adaptabilidade e estabilidade foram a população CNPH-15 e a cultivar Juliana. A população CNPH-16 e a cultivar Brasília apresentaram resultados intermediários.

A informação de que a cultivar BRS Planalto apresenta boa adaptabilidade e estabilidade é concordante com Silva et al. (2012), que estudaram esta cultivar além da cultivar Brasília e outras duas populações do grupo Brasília em cinco ambientes, estimando os valores genotípicos, e verificaram que BRS Planalto apresentou a melhor adaptabilidade e estabilidade.

O fato de que a cultivar Brasília apresentou resultados intermediários quanto à adaptabilidade e estabilidade concorda com a literatura. Estudando a adaptabilidade e estabilidade da cultivar Brasília em regiões dos estados de São Paulo, Minas Gerais e Paraná, Oliveira et al. (2005) verificaram que esta cultivar respondeu medianamente a ambientes favoráveis e se destacou pela tolerância à queima-das-folhas. $\mathrm{O}$ mesmo foi verificado por Oliveira et al. (2008) que avaliaram esta mesma cultivar em várias épocas e espaçamentos de semeadura em São José do Rio Pardo-SP. Silva et al. (2012) também verificaram que a cultivar Brasília, avaliada em cinco ambientes, não teve adaptação ampla, mas específica a Lapão. Pôde-se verificar que as populações com melhor desempenho e adaptabilidade e estabilidade nos ambientes estudados foram BRS Planalto e as populações CNPH-14 e CNPH-17; a cultivar Juliana e a população CNPH-15 apresentaram os piores resultados frente à média dos ambientes, e Juliana apresentou adaptação específica a São Gotardo e Canoinhas.

\section{REFERÊNCIAS}

ANUÁRIO BRASILEIRO DE HORTALIÇAS.
2015. Brazilian Vegetable Yearbook. Santa Cruz do Sul: Gazeta, 68p.

CAMPBELL, BT; JONES, MA. 2005. Assessment of genotype $\mathrm{x}$ environment interactions for yield and fiber quality in cotton performance trials. Euphytica 144: 69-78.

CARVALHO, AM; JUNQUEIRA, AMR; VIEIRA, JV; REIS, A; SILVA, JBC. 2005. Produtividade, florescimento prematuro e queima-das-folhas em cenoura cultivada em sistema orgânico e convencional. Horticultura Brasileira 23: 250-254.

CEAGESP. 1999. Classificação de cenoura: programa de adesão voluntária (Folder), São Paulo: Programa Horti \& Fruti. 8p.

CRUZ, CD; REGAZZI, AJ; CARNEIRO, PCS. 2012. Modelos biométricos aplicados ao melhoramento genético. 4.ed. Viçosa: UFV. $514 \mathrm{p}$.

DUARTE, JB; ZIMMERMAN, MJO. 1994. Adaptabilidade e estabilidade de rendimento de populações de feijão comum. Pesquisa Agropecuária Brasileira 29: 25-32.

EBERHART, SA; RUSSELL, WA. 1966. Stability parameters for comparing varieties. Crop Science 6: 36-40.

HENDERSON, CR. 1975. Best linear estimation and prediction under a selection model. Biometrics 31: 423-447.

LIN, CS; BINNS, MR. 1988. A method for analyzing cultivar $\mathrm{x}$ location $\mathrm{x}$ years experiments: a new stability parameter. Theoretical and Applied Genetics 76: 425-430.

NAMORATO, H; MIRANDA, GV; SOUZA, LV; OLIVEIRA, LR; DELIMA, RO; MANTOVANI, EE. 2009. Comparing biplot multivariate analyses with Eberhart and Russel' method for genotype x environment interaction. Crop Breeding and Applied Biotechnology 9: 299-307.

OLIVEIRA, CD; BRAZ, LT; BANZATTO, DA. 2005. Adaptabilidade e estabilidade genotípica de populações de cenoura. Horticultura Brasileira 23: 743-748.

OLIVEIRA, CD; BRAZ, LT; BANZATTO, DA. 2008. Adaptabilidade e estabilidade fenotípica de cultivares de cenoura. Horticultura Brasileira 26: 88-92.

OSMANZAI, M; SHARMA, RC. 2008. High yielding stable wheat genotypes for the diverse environments in Afghanistan. International Journal of Agricultural Research 3: 340-348.

RESENDE, MDV. 2002a. Genética biométrica e estatística no melhoramento de plantas perenes. Brasília: Embrapa Informação Tecnológica. 975p.

RESENDE, MDV. 2002b. Software SelegenREML/BLUP. Curitiba: Embrapa Florestas, $67 \mathrm{p}$.

RESENDE, GM; BRAGA, MB. 2014. Produtividade de cultivares e populações de cenoura em sistema orgânico de cultivo. Horticultura Brasileira 32: 102-106.

RESENDE, GM; CORDEIRO, GG. 2007. Produtividade da cenoura em função da qualidade da água e condicionador de solo no Vale do São Francisco. Caatinga 20: 100-104.

SILVA, GO; CARVALHO, ADF; VIEIRA, JV; BENIN, G. 2011. Verificação da adaptabilidade 
e estabilidade de populações de cenoura pelos métodos AMMI, GGE biplot e REML/BLUP. Bragantia 70: 494-501.

SILVA, GO; CARVALHO, ADF; VIEIRA, JV; FRITSCHE-NETO, R. 2012. Adaptability and stability of the carrot population. Horticultura Brasileira 30: 80-83.

SOARES, IAA; FREITAS, FCL; NEGREIROS, MZ; FREIRE, GM; AROUCHA, EMM; GRANGEIRO, LC; LOPES, WAR;
DOMBROSKI, JLD. 2010. Interferência das plantas daninhas sobre a produtividade e qualidade de cenoura. Planta daninha 28: 247-254.

SOUZA, JL. 1996. Efeito do composto orgânico e da cobertura morta na cultura da cenoura. In: CONGRESSO BRASILEIRO DE OLERICULTURA, 36. Resumos... Rio de Janeiro: SOB. v.14.

VIEIRA, JV; SILVA, GO. 2008. Tamanho mínimo de parcela para avaliação de caracteres de raiz em cenoura. Bragantia 67: 817-825.

VIEIRA, JV; SILVA, GO; BOITEUX, LS; SIMON, P. 2009. Divergência genética entre acessos de cenoura pertencentes a grupos varietais distintos utilizando caracteres morfológicos. Horticultura Brasileira 27: 468-472.

WRICKE, G. 1965. Zur Berechnung der Ökovalenz bei Sommerweizen und Hafer. Pflanzenzüchtung 52: 127-138. 